\title{
A Generalized Error Distribution-based method for Conditional Value-at-Risk evaluation
}

\author{
Roy Cerqueti, Massimiliano Giacalone, and Demetrio Panarello
}

\begin{abstract}
One of the most important issues in finance is to correctly measure the risk profile of a portfolio, which is fundamental to take optimal decisions on the capital allocation. In this paper, we deal with the evaluation of portfolio's Conditional Value-at-Risk (CVaR) using a modified Gaussian Copula, where the correlation coefficient is replaced by a generalization of it, obtained as the correlation parameter of a bivariate Generalized Error Distribution (G.E.D.). We present an algorithm with the aim of verifying the performance of the G.E.D. method over the classical RiskMetrics one, resulting in higher performance of the G.E.D. method.
\end{abstract}

\section{Introduction}

Value-at-Risk (VaR) has become a standard measurement tool in financial risk management due to its simplicity. However, it is an unstable and numerically difficult to use method when the losses do not follow a Gaussian distribution (Ferraty \& Quintela-Del-Río, 2016), which is usually the case in the analysis of financial data. Conditional VaR (CVaR, see Rockafellar \& Uryasev, 2001; Acerbi \& Tasche, 2002; Huang et al., 2010) has been proposed by literature as an alternative to VaR (Artzner et al., 1999). For a better calculation of the risk, one of the proposals (e.g. Malevergne \& Sornette, 2003) is to model the interdependence of the returns by means of Copula functions (see McNeil et al., 2015). In this context, the problem can be split

Roy Cerqueti

Department of Economics and Law, University of Macerata e-mail: roy.cerqueti@unimc.it

Massimiliano Giacalone

Department of Economics and Statistics, University of Naples 'Federico II' e-mail: massimiliano.giacalone@unina.it

Demetrio Panarello

Department of Economic and Legal Studies, Parthenope University of Naples e-mail: demetrio.panarello@uniparthenope.it 
into two separate parts: first, to identify the marginal distributions of the returns of the single assets; second, to identify the specific copula which is more appropriate for representing the dependence structure of the returns (see Sklar, 1959).

In Section 2, we introduce the quantitative ingredients of the study, with the main definitions. The proposed methodological setting for calculating the Conditional Value-at-Risk of a generic portfolio is presented in Section 3. Finally, in Section 4 some conclusive remarks are given.

\section{The Generalized Error Distribution and the G.E.D. Copula}

The Generalized Error Distribution (G.E.D.) family was introduced by Subbotin (1923) and has been employed by various authors with different names and parameterizations (see e.g. Box \& Tiao, 1973; Chiodi, 1986; Agrò, 1999; Mineo, 2007; Bottazzi \& Secchi, 2011). A parameterization of the G.E.D. density function for a random variable $X$ is:

$$
f\left(x ; \mu, \sigma_{p}, p\right)=\frac{1}{2 \sigma_{p} p^{1 /(p)} \Gamma(1+1 / p)} \exp \left(-\frac{1}{p}\left|\frac{x-\mu}{\sigma_{p}}\right|^{p}\right) \text { for }-\infty<\mathrm{x}<\infty
$$

where $\mu=E(X)$ is the location parameter, $\sigma_{p}=\left[E|X-\mu|^{p}\right]^{1 / p}>0$ is the scale parameter, $p>0$ is the shape parameter and $\Gamma$ is the Euler Gamma function.

The density of a generic G.E.D. distribution is unimodal, symmetric and, for $p>$ 1, bell-shaped. As particular cases we obtain the Laplace distribution $(p=1)$, the Normal $(p=2)$ and the Uniform $(p \rightarrow \infty)$; for values of $1<p<2$ we obtain leptokurtic densities and for values of $p>2$ we obtain platikurtic densities. Thus, the G.E.D. represents a generalization of a large set of distributions, allowing for a better description of financial data.

A bivariate copula is a function $C:[0,1]^{2} \rightarrow[0,1]$ whose main interest in the field of probability is that it associates univariate marginal distributions to their joint ones (Sklar, 1959). We are here interested in the bivariate Gaussian Copula, which is defined as:

$$
C(u, v \mid \rho)=\int_{-\infty}^{\Phi^{-1}(u)} \int_{-\infty}^{\Phi^{-1}(v)} \frac{1}{2 \pi \sqrt{\left(1-\rho^{2}\right)}} \exp \left\{\frac{-\left(r^{2}-2 \rho r s+s^{2}\right)}{2\left(1-\rho^{2}\right)}\right\} d r d s,
$$

where $\Phi^{-1}$ is the inverse of Gaussian distribution function, $(u, v)$ uniform independent random variables generated from $(\mathrm{X}, \mathrm{Y})$ random variables, and $\rho \in[-1,1]$ is a parameter representing the Pearson's correlation coefficient associated to the bivariate normal. The G.E.D. Copula for a generic random vector $(X, Y)$ is obtained by replacing the parameter $\rho$ by the Generalized Correlation Coefficient $\rho_{p} \in[-1,1]$, introduced by Taguchi (1974) and estimated as follows:

$$
\rho_{p}=\frac{\operatorname{codisp}^{(p)}(X, Y)}{\sigma_{p}(X) \sigma_{p}(Y)}, \text { with }-1 \leq \rho_{\mathrm{p}} \leq 1,
$$


where

$$
\begin{gathered}
\left|\operatorname{codisp}^{(p)}(X, Y)\right|^{p}=\left|E\left[\left(Y-\mu_{Y}\right)\left|X-\mu_{X}\right|^{p-1} \operatorname{sign}\left(X-\mu_{X}\right)\right]\right| \cdot\left|E\left[\left(X-\mu_{X}\right)\left|Y-\mu_{Y}\right|^{p-1} \operatorname{sign}\left(Y-\mu_{Y}\right)\right]\right|, \\
\sigma_{p}(X)=\left[E\left|X-\mu_{X}\right|^{p}\right]^{1 / p}, \quad \sigma_{p}(Y)=\left[E\left|Y-\mu_{Y}\right|^{p}\right]^{1 / p}
\end{gathered}
$$

and $\mu_{X}$ and $\mu_{Y}$ are the expected values of $X$ and $Y$, respectively. The parameters $\mu, p$ and $\sigma_{p}$ could be estimated e.g. by using the $L p_{\min }$ method (Giacalone, 1996; Giacalone \& Richiusa, 2006).

\section{The methodology}

We here discuss theoretically how to compute the CVaR in a G.E.D. framework. Real world applications on financial data are available upon request.

We start from two sets of consecutive observations of the returns of two assets, assumed to be empirically distributed according to a G.E.D.:

$$
X=\left\{x_{1}, \ldots, x_{n}\right\}, \quad Y=\left\{y_{1}, \ldots, y_{n}\right\}
$$

For what concerns the stochastic dependence between $X$ and $Y$, we propose a G.E.D. copula model.

The fundamental steps in the algorithm for the computation of the CVaR of a portfolio consisting of $(\alpha, \beta)$ are as follows:

0 . we fix a portfolio $(\alpha, \beta)$, with $\alpha+\beta=1$;

1. estimation of the parameters $\mu, p, \sigma_{p}$ in (1) for the two series of returns $X$ and $Y$. In accord to the notation used above, we will denote the parameters as $\mu_{X}, p_{X}, \sigma_{p, X}$ and $\mu_{Y}, p_{Y}, \sigma_{p, Y}$

2. estimation of the $\rho_{p}$ parameter of the G.E.D. copula by using formula (3), with $p=\alpha p_{X}+\beta p_{Y}$

3. generation of couples $(x, y)$, which are the realization of the double stochastic variable $(X, Y)$ having G.E.D. marginals of item 1. and stochastic dependence described by the G.E.D. copula with $\rho_{p}$ of item 2 ;

4. construction of the realizations of the returns of portfolio $P=\alpha X+\beta Y$ and of its empirical distribution;

5. computation of the Value-at-Risk of $P$ at a confidence level $(1-c) \%$;

6. computation of the Conditional Value-at-Risk of $P$ at a confidence level $(1-c) \%$.

The algorithm above is repeated for all the considered portfolios, given by $\alpha=$ $0.01: 0.01: 0.99$ and $\beta=1-\alpha$. 


\section{Conclusions}

The present paper is part of market risk calculation methods, whose purpose is to support risk managers' decision making processes. Among the different methods proposed in the literature for calculating Value-at-Risk, we took the well-known RiskMetrics into account. We introduced the new G.E.D. method and proposed the G.E.D. Copula as a generalization of the Gaussian Copula. Moreover, we introduced the Generalized Correlation Coefficient of norm $\mathrm{p}$ that, for the $p=2$ case, equals the classic Bravais-Pearson correlation coefficient. We then presented an algorithm with the aim of verifying the performance of the new method over the classical RiskMetrics one. The problem of whether CVaR-G.E.D. can constitute a valid generalization of CVaR-R.M. is still debatable, and an empirical analysis would provide more insights on this relevant topic.

\section{References}

1. Acerbi, C., \& Tasche, D. (2002). On the coherence of expected shortfall. Journal of Banking \& Finance, 26(7), 1487-1503.

2. Agrò, G. (1999). Parameter orthogonality and Conditional Profile Likelihood: the Exponential Power Function Case. Commun.Statist.-Theory Meth., 28(8), 1759-1768.

3. Artzner, P., Delbaen, F., Eber, J. M., \& Heath, D. (1999). Coherent measures of risk. Mathematical Finance, 9(3), 203-228.

4. Bottazzi, G., \& Secchi, A. (2011). A new class of asymmetric exponential power densities with applications to economics and finance. Industrial and Corporate Change.

5. Box, G. E., \& Tiao, G. C. (1973). Bayesian inference in statistical analysis. Addison-Wesley.

6. Chiodi, M. (1986). Procedures for Generating Pseudo-Random Numbers from a Normal Distribution of Order p. Riv.Stat.Applicata, 19, 7-26.

7. Ferraty, F., \& Quintela-Del-Río, A. (2016). Conditional VaR and expected shortfall: a new functional approach. Econometric Reviews, 35(2), 263-292.

8. Giacalone, M. (1996). Parameter evaluation of an exponential power function by simulation study. In Shorts Communications and Posters, Compstat 96, Barcelona.

9. Giacalone, M., \& Richiusa, R. (2006). Lp-norm estimation: some simulation studies in presence of multicollinearity. Student, 5, 235-246.

10. Huang, D., Zhu, S., Fabozzi, F. J., \& Fukushima, M. (2010). Portfolio selection under distributional uncertainty: A relative robust CVaR approach. European Journal of Operational Research, 203(1), 185-194.

11. Malevergne, Y., \& Sornette, D. (2003). Testing the Gaussian Copula hypothesis for financial assets dependences. Quantitative Finance, 3, 231-250.

12. McNeil, A. J., Frey, R., \& Embrechts, P. (2015). Quantitative risk management: Concepts, techniques and tools. Princeton University Press.

13. Mineo, A. M. (2007). On the estimation of the structure parameter of a normal distribution of order p. Statistica, 63(1), 109-122.

14. Rockafellar, R. T., \& Uryasev, S. (2002). Conditional value-at-risk for general loss distributions. Journal of Banking \& Finance, 26(7), 1443-1471.

15. Sklar, M. (1959). Fonctions de répartition à $\mathrm{n}$ dimensions et leurs marges. Université Paris 8 .

16. Subbotin, M. (1923). On the Law of Frequency of Error. Mathematicheskii Sbornik.

17. Taguchi, T. (1974). On Fechner's thesis and statistics with Norm-p. Annals of the Institute of Statistical Mathematics, 26(1), 175-193. 